\title{
Determination of the amount of leg length inequality that alters spinal posture in healthy subjects using rasterstereography
}

\author{
Marcel Betsch • Walter Rapp • Anna Przibylla • \\ Pascal Jungbluth $\cdot$ Mohssen Hakimi $\cdot$ Johannes Schneppendahl • \\ Simon Thelen $\cdot$ Michael Wild
}

Received: 25 August 2012/Revised: 19 December 2012/Accepted: 12 February 2013/Published online: 13 March 2013

(C) Springer-Verlag Berlin Heidelberg 2013

\begin{abstract}
Purpose Leg length inequalities (LLIs) can result in an increased energy consumption, abnormal gait or osteoarthritis of the hip. In a previous study we simulated different LLIs of up to $15 \mathrm{~mm}$ and evaluated their effects on the pelvic position and spinal posture. We found a correlation between LLIs and resulting changes of the pelvic position. Despite suggestions in the literature we were not able to detect significant changes of the spinal posture. Therefore, the purpose of this study was to determine the amount of LLI that would in fact alter the spinal posture.

Methods The subjects were placed on a simulation platform, whose height could be precisely controlled by the measuring device, to simulate different LLIs of up to $20 \mathrm{~mm}$. For LLIs $>20 \mathrm{~mm}$, additional precision-cut wooden blocks were used under one foot. After an adaptation period the resulting changes of the pelvis and spine were measured with a rasterstereographic device.
\end{abstract}

M. Betsch · A. Przibylla · P. Jungbluth · M. Hakimi ·

J. Schneppendahl $\cdot$ S. Thelen

Department of Trauma and Hand Surgery, University Hospital

Duesseldorf, Duesseldorf, Germany

M. Betsch ( $\square)$

Department of Orthopaedics and Rehabilitation, Oregon Health and Science University, 3181 S.W. Sam Jackson Park Rd., Portland, OR 97239-3098, USA

e-mail: betsch@ohsu.edu

W. Rapp

Department of Sports Medicine, University Hospital Tuebingen, Tuebingen, Germany

M. Wild

Department of Trauma and Orthopaedic Surgery,

Klinikum Darmstadt, Darmstadt, Germany
Results We found a significant correlation between platform height changes and changes of the pelvic position. The frontal spinal parameters surface rotation and lateral deviation changed significantly when simulating differences greater than $20 \mathrm{~mm}$. No changes of the sagittal spinal curvature were measured, however, a trend to decreasing kyphotic angles was noted.

Conclusions Our study has shown for the first time that LLIs $>20 \mathrm{~mm}$ will lead to significant changes in the spinal posture of healthy test subjects. However, these changes were only found in frontal (surface rotation and lateral flexion) spinal parameters, but not in sagittal parameters. Here for the kyphotic angle only a tendency to decreasing angles was noted. We have also found a significant correlation between different leg lengths and changes of the pelvic position. Further, females and males seem to react in the same way to LLIs.

Keywords Leg length inequalities - Rasterstereography · Posture $\cdot$ Pelvic obliquity $\cdot$ Functional scoliosis

\section{Introduction}

Differences in the leg length can be due to height differences of the foot, tibia, femur or the pelvis. But the total length difference in the standing position is not only due to the involved bones, it can also be altered by anatomic abnormalities such as unilateral joint dislocation, subluxation or contractures of the hip, knee or ankle joint, as well as by fixed pelvic obliquity. The compensation for leg length differences in the lower extremity, the pelvis and spine can lead to muscular imbalances, which may result in iliosacral and low back pain [1]. 
The correlation between leg length differences and compensatory lumbar scoliosis has been studied with various systems, making it difficult to compare their results [2-4]. In a previous experimental study we simulated different leg lengths of up to $15 \mathrm{~mm}$ and their effects on the pelvis and spinal posture [5]. We found a correlation between simulated leg length differences and resulting changes of the pelvic position. Despite suggestions in the literature, we were not able to detect any significant changes of the spinal posture when simulating leg length inequalities (LLIs) up to $15 \mathrm{~mm}$. Therefore, in this present study we simulated LLIs of up to $60 \mathrm{~mm}$ to find the point at which such discrepancies do in fact alter spinal posture.

\section{Materials and methods}

We examined 100 volunteers (53 females and 47 males) with a mean age of 34 years and a mean height of $173 \mathrm{~cm}$ for this study. We included only healthy test subjects without any history of a fracture or spinal abnormality. Volunteers with a pre-existing pelvic obliquity greater than $10 \mathrm{~mm}$, any history of back pain longer than 2 days in the previous year, and a body mass index (BMI) greater than $35 \mathrm{~kg} / \mathrm{m}^{2}$ were excluded from this study. The study was approved by the human subjects research review board and all subjects were informed about the study, gave their written consent and were given the option to discontinue participation at any time.

Spinal posture and pelvic position were measured with the Formetric ${ }^{\circledR}$ rasterstereography system (Diers International GmbH, Schlangenbad, Germany). Rasterstereography is a method for stereophotogrammetric surface measurement of the back, which was developed in the 1980s by Hierholzer and Drerup [6]. Horizontal parallel light lines are projected onto the unclothed surface of the back by a slide projector. A surface reconstruction of the back is performed, by transforming the lines and their corresponding curvature into a three-dimensional scatter plot. A 3D-model of the spine can then be calculated based on the specific convex shape of the spinous process of the vertebra prominence (VP) and the concavity of the lumbar dimples. Transverse and sagittal profiles, the spinous process line and several spinal angles and indices can be calculated with this model. It is possible to use the two lumbar dimples to determine pelvic obliquity, because they are in close relation to the underlying posterior superior iliac spines [6]. From the orientation of the skin surface over the lumbar dimples it is also feasible to draw conclusions about the pelvic torsion around the transverse axis [6, 7].

The subjects were placed on a simulation platform, whose height could be precisely controlled by the measuring device (Fig. 1). For LLIs greater than $20 \mathrm{~mm}$, additional precision-cut wooden blocks were placed under one foot. The weight distribution between both legs was measured by the simulation platform to ensure an equal weight distribution. All subjects stood for $60 \mathrm{~s}$ to adapt to the simulated LLI (leg length inequality) prior to the measurement. The recordings were performed with the subjects standing in a relaxed posture with extended knees on the platform, which is considered the neutral position. The following LLIs (right then left leg) were simulated: $+0,+10,+20,+30,+40,+50$ and $+60 \mathrm{~mm}$. In pre-tests we determined a maximum height for the simulated LLIs of $60 \mathrm{~mm}$, because with greater heights a full extension of the knee joint was no longer possible.

For the purpose of this study it is necessary to define certain terms regarding the parameters that were measured. The pelvic obliquity is the amount of tilt in degrees or millimetres from the horizontal line between the two lumbar dimples DL (left dimple) to DR (right dimple) (Fig. 2). A positive value indicates that the right dimple is higher than the left and a negative value indicates that the left dimple is higher than the right. The pelvic torsion measured in degrees is the rotation of the surface normals of the two lumbar dimples (DL and DR) as shown in Fig. 2. A positive pelvic torsion means that the right hipbone is oriented farther anterior than the left hipbone and a negative value signifies that the left hipbone is farther anterior than the right hipbone. The pelvic inclination is defined as the mean vertical torsion of the two surface normals on the two lumbar dimples. Figure 3 illustrates the surface rotation as the value of the horizontal components of the surface normals on the line of symmetry (line connecting the spinous processes of the spine) measured in degrees $\left({ }^{\circ}\right)$. The lateral deviation is defined as the deviation

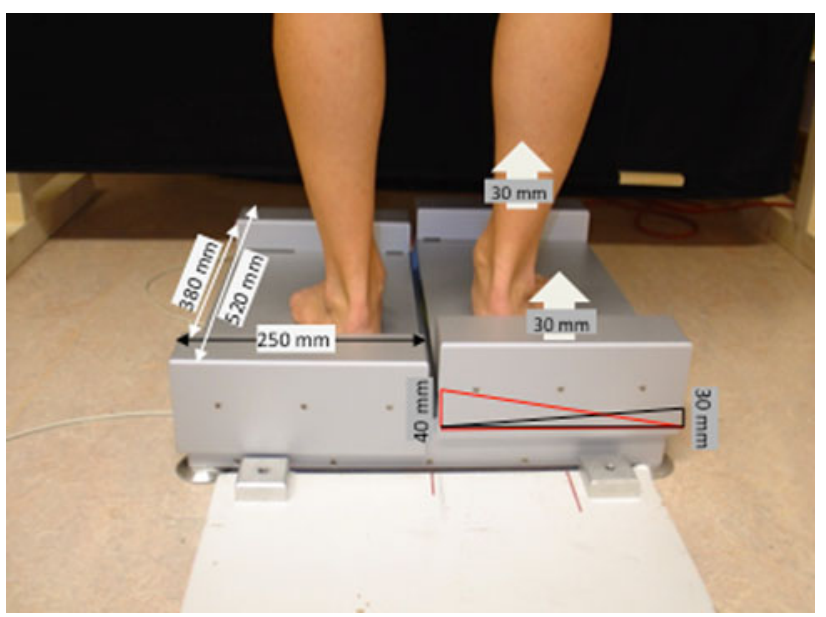

Fig. 1 The figure shows a test subject standing on the stand platform, which is raised $20 \mathrm{~mm}$ on the right side, during a rasterstereographic measurement. The specific dimensions of the constructed platform are included into the figure. When simulating LLIs greater than $20 \mathrm{~mm}$ additional precision-cut wooden blocks were placed under the foot 


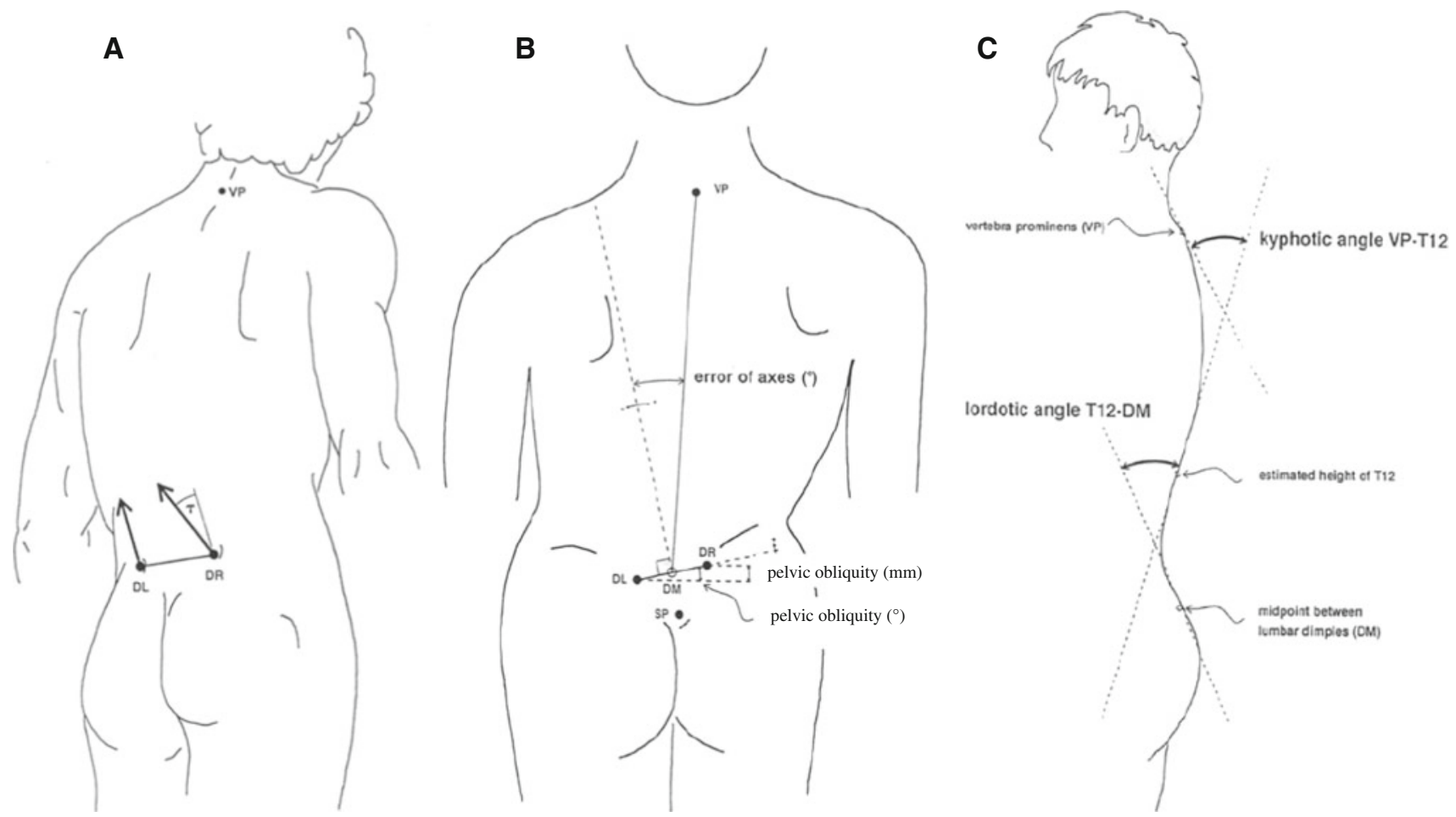

Fig. 2 Pelvic torsion as shown in a is defined as the torsion of the surface normals on the two lumbar dimples [left lumbar dimple (DL) and right lumbar dimple (DR)]. b Illustrates the pelvic obliquity, which is considered as the different height of the two lumbar dimples to each other in degrees. In $\mathbf{c}$ the kyphotic angle is illustrated as the

of the spinal midline from the line between the VP to midpoint between DL and DR (DM) in the frontal plane (Fig. 3). The kyphotic angle is the angle between the surface tangents on points VP and the calculated spinous process of the 12th thoracic vertebrae (T12) and the lordotic angle is the angle between the surface tangents on points T12 and DM (Fig. 2).

Data analysis

All data were checked for Gaussian distribution by the Chisquare test and presented as means with standard deviations or $95 \%$ confidence level. Unifactorial ANOVA was calculated to check for changes in the values. The level of significance was set at $p<0.05$. Correlation between the measured parameters was calculated using the Pearson correlation. Statistical analysis and graphic presentation were prepared using software SPSS $20.0^{\circledR}$ (SPSS Inc., Chicago, USA).

\section{Results}

In the neutral standing position we measured a mean pelvic obliquity of $4.23 \mathrm{~mm} \pm 4.23 \mathrm{~mm}$ (mean $\pm \mathrm{SD}$ ). There angle between the surface tangents on spinous process of the seventh cervical vertebra (VP) and the calculated spinous process of the 12th thoracic vertebrae (T12). The lordotic angle was measured as the angle between the surface tangents on points T12 and the midpoint between the two lumbar dimples (DM)

was no significant difference between the neutral pelvic position of females and males. Furthermore, there were no gender differences in response to the pelvic obliquity to the LLI. Inducing a LLI led to an increase of the pelvic obliquity on the corresponding side (Fig. 4). All LLIs greater than $20 \mathrm{~mm}$, left and right leg $(p<0.05)$, led to significant changes of the pelvic obliquity. The mean pelvic torsion with $0 \mathrm{~mm}$ LLI was $1.54^{\circ} \pm 1.05^{\circ}$ for women and $2.36^{\circ} \pm 1.61^{\circ}$ for men. Figure 4 illustrates that with increasing LLIs an increase in the pelvic torsion occurred. We did not find differences in response to the LLIs for pelvic torsion between females and males. Simulated LLIs of $20 \mathrm{~mm}$ on the right and $30 \mathrm{~mm}$ on the left side led to significant changes in the pelvic torsion. There was a significant correlation between the platform height and the parameters pelvic obliquity $(r=0.894)$ and torsion $(r=0.741)$, as well as between pelvic obliquity and pelvic torsion $(r=0.639)$. The mean pelvic inclination in the neutral standing position was $20.95^{\circ} \pm 6.03^{\circ}$ for women and $13.12^{\circ} \pm 5.77^{\circ}$ for men. In the neutral standing position as well as in response to all simulated LLIs, there was a significant difference $(p<0.05)$ measured between women and men for pelvic inclination. Increasing the simulated leg length differences led to an increase in pelvic inclination (Fig. 5). These changes in pelvic inclination 
Correlation Model Surface to Spine by Turner/Smith\&Drerup

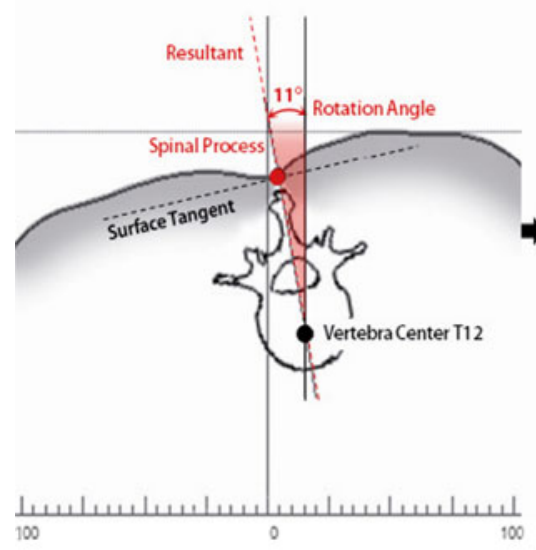

A

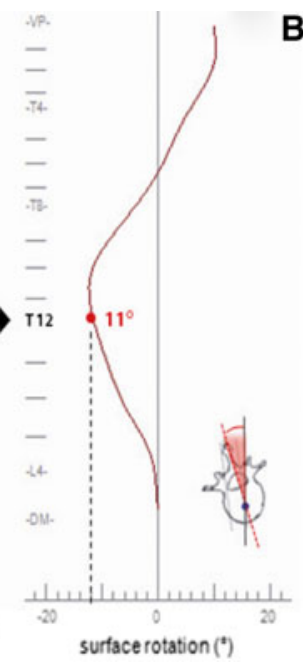

Fig. 3 a, b Illustrate the surface rotation as the value of the horizontal components of the surface normals on the line of symmetry measured in degrees. The lateral deviation is defined as the deviation

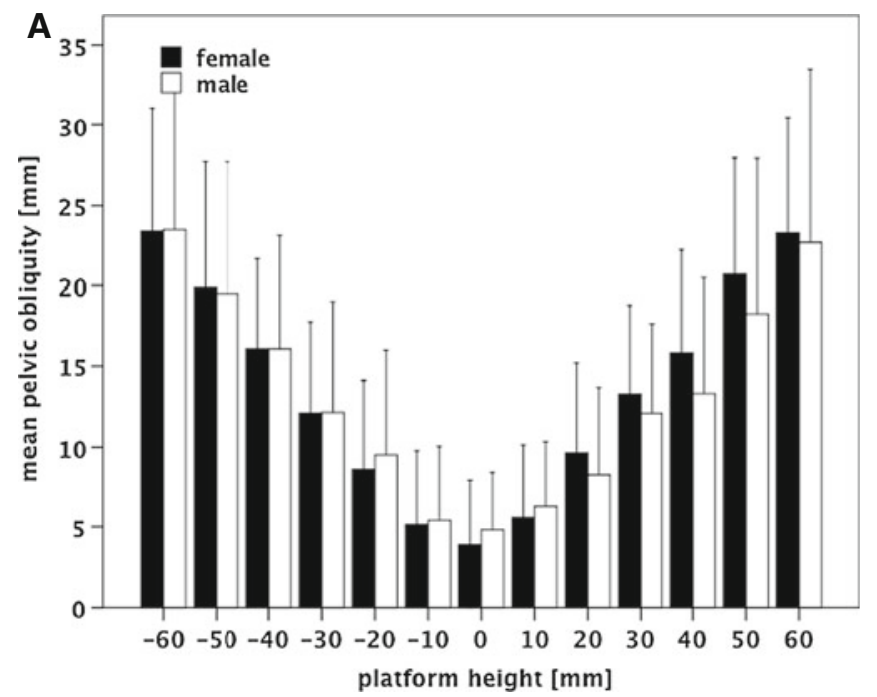

Fig. 4 a Shows the mean pelvic obliquity in millimetres (with the $95 \%$ confidence level) for the simulated different platform heights $(0-60 \mathrm{~mm})$ for the right side (positive values) and left side (negative values). An increase in platform height leads to an increase in pelvic obliquity on the corresponding side. b Demonstrates the changes of

were significant ( $p<0.05$ ), for LLIs of $50 \mathrm{~mm}$ and above, when compared to the neutral standing position.

The aim of this study was to determine the amount of LLI that will lead to changes in the spinal posture. A parameter that describes the frontal orientation of the spine is the surface rotation, which was measured with a mean value of $3.73^{\circ} \pm 1.72^{\circ}$. Inducing LLIs greater than $30 \mathrm{~mm}$ on the right and $20 \mathrm{~mm}$ on the left side $(p<0.05)$ led to significant changes in the surface rotation (Fig. 6). No differences were measured between females $\left(3.78^{\circ} \pm 1.86^{\circ}\right)$ and males

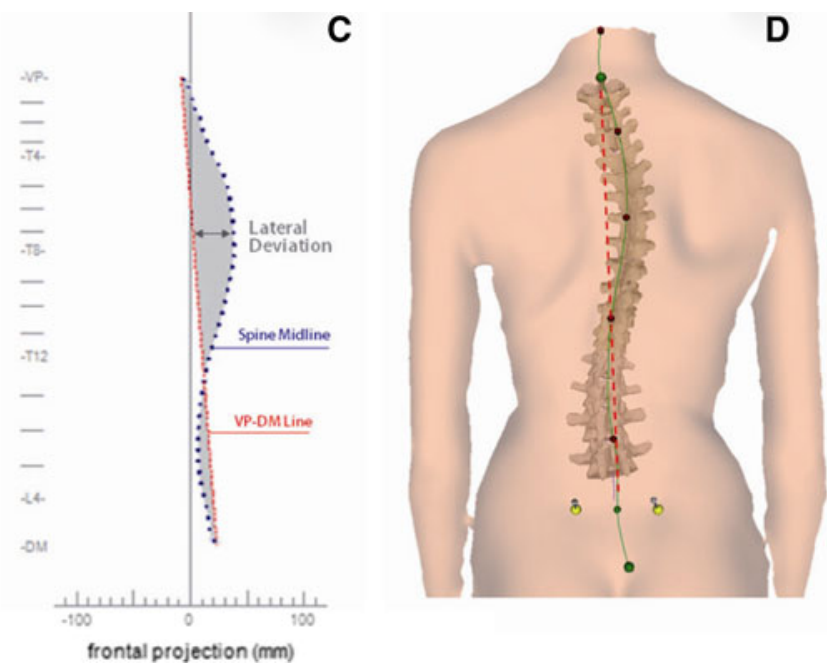

of the spinal midline from the line between the VP to DM (midpoint between DL and DR) as shown in $\mathbf{c}$ and $\mathbf{d}$

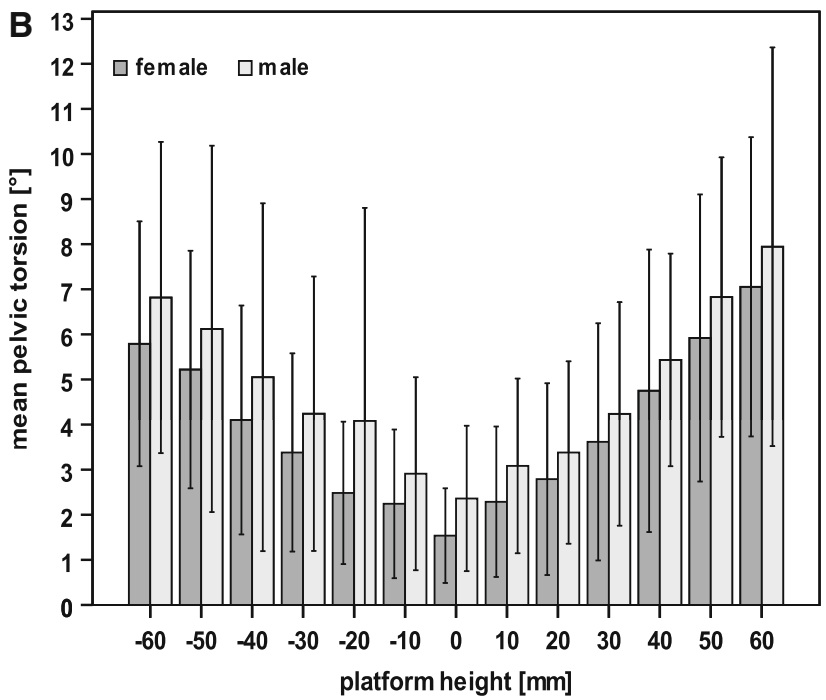

the pelvic torsion in degrees with the simulated platform heights of up to $60 \mathrm{~mm}$. The changes in pelvic torsion are significant between the neutral position $(0 \mathrm{~mm})$ and platform heights $\geq 20 \mathrm{~mm}$ on the right side and $\geq 30 \mathrm{~mm}$ on the left side. No significant differences were found between females and males

$\left(3.67^{\circ} \pm 1.47^{\circ}\right)$ for this parameter. We found a significant correlation between the surface rotation and the platform height $(r=-0.112)$ as well as the pelvic obliquity $(r=-0.112)$.

A mean value of $4.70 \pm 3.12 \mathrm{~mm}$ for the lateral deviation was determined. We found an increase in the lateral deviation with increasing LLIs in both legs (Fig. 6). The increase in lateral deviation was significant with LLIs greater than $30 \mathrm{~mm}$ on the right and $20 \mathrm{~mm}$ on the left leg. In addition the results of the measurements showed that the spine deviated always towards the short leg side (Fig. 7). 
Further, we found a significant correlation between the parameter lateral deviation and the platform height $(r=$ $-0.141)$ and pelvic obliquity $(r=-0.167)$. No differences were found between females $\left(48.94^{\circ} \pm 8.51^{\circ}\right)$ and males $\left(51.97^{\circ} \pm 10.26^{\circ}\right)$ for the kyphotic angle (Table 1). The simulated LLIs did not create significant changes in the kyphotic angle, however, we found a tendency to decreasing kyphotic angles with increasing LLIs. Generally, females had a significant higher lordosis $41.96^{\circ} \pm$ $8.42^{\circ}$ than males $33.52^{\circ} \pm 8.04^{\circ}$ (Table 1 ). Finally, we did not find any significant differences in the lordotic angles when increasing the LLI.

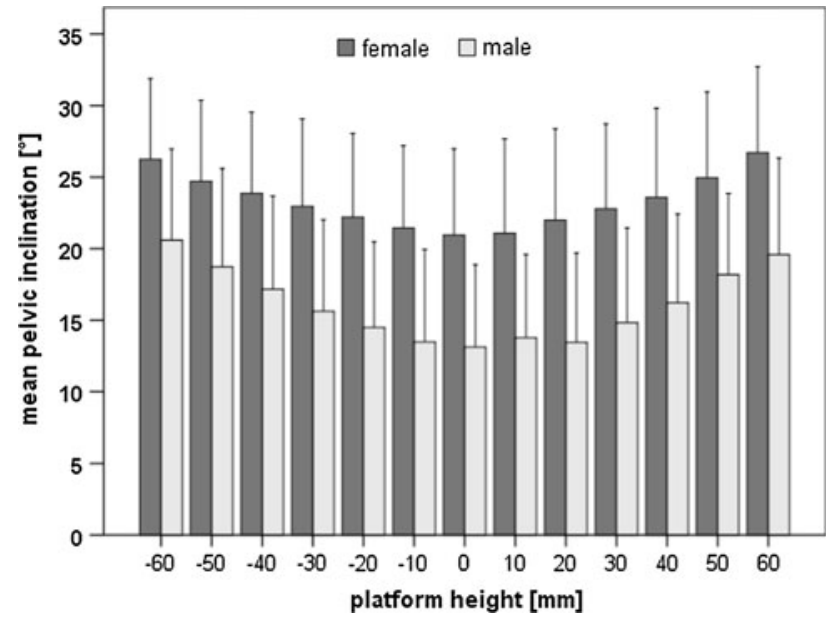

Fig. 5 The increase in LLI led to an increase in pelvic obliquity. A significant increase was found on both sides with LLIs of $50 \mathrm{~mm}$ and above $(p<0.05)$. We measured a significant difference $(p<0.05)$ in the neutral standing position and in all simulated leg lengths between women and men

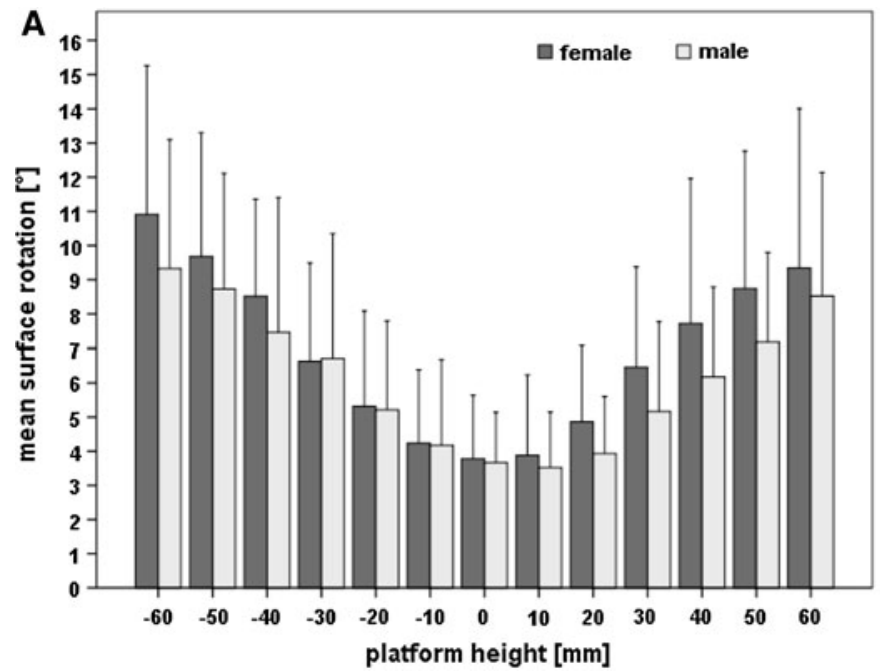

Fig. 6 a Shows the changes of the frontal parameter surface rotation (with the $95 \%$ confidence level) when raising the platform. Changes between the neutral position ( $0 \mathrm{~mm}$ platform height) and $\geq 20 \mathrm{~mm}$ on the left side and $\geq 30 \mathrm{~mm}$ on the right side are significant. b Shows

\section{Discussion}

The purpose of the present study was to determine the amount of LLI that will create changes in spinal posture. Similar to Beaudoin et al. [8], we have focused on changes of the spine and pelvis due to LLIs, because such changes may lead to musculoskeletal disorders and possibly to functional scoliosis. Previous studies have investigated the effects of LLIs on the lower extremity $[9,10]$, but to the best of our knowledge no one has tried to determine the amount of LLI that will directly lead to changes in spinal parameters.

We decided to use a rasterstereographic system, because it allows a radiation-free analysis of spinal posture and pelvic position. Multiple studies have shown the high accuracy and reliability of this system [11, 12]. Hackenberg et al. [13] compared radiographs with rasterstereographic measurements and found a good correlation of $r=0.89$ for the frontal deviation in subjects even with scoliosis. Good reliability was also found by Goh [14], who analysed healthy subjects. Further, Crawford et al. [15] and Goh et al. [16] could show a high correlation between rasterstereographically and radiologically measured kyphotic and lordotic angles. A previous study has shown the strong correlation between the posterior superior iliac spines and the left and right dimple [17]. Due to the high accuracy and validity as demonstrated in these studies, we used video rasterstereography to determine the position of the lumbar dimples and the underlying pelvis.

In a previously published study we investigated the effects of minor LLIs up to $15 \mathrm{~mm}$ on the pelvis and spine [5]. We found a strong correlation between LLIs and

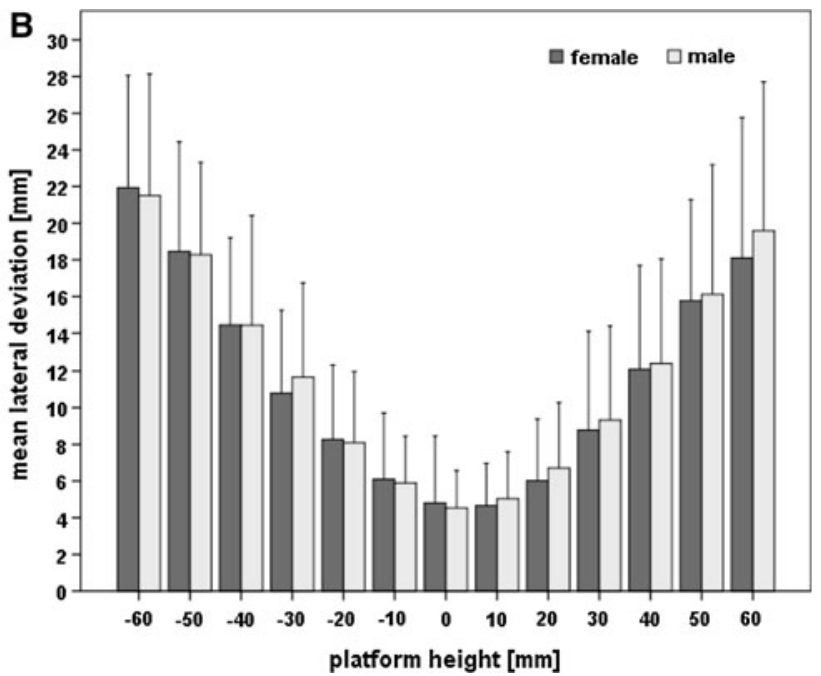

the increase of the lateral deviation of the spine, when increasing the platform height. No differences between females and males were found 


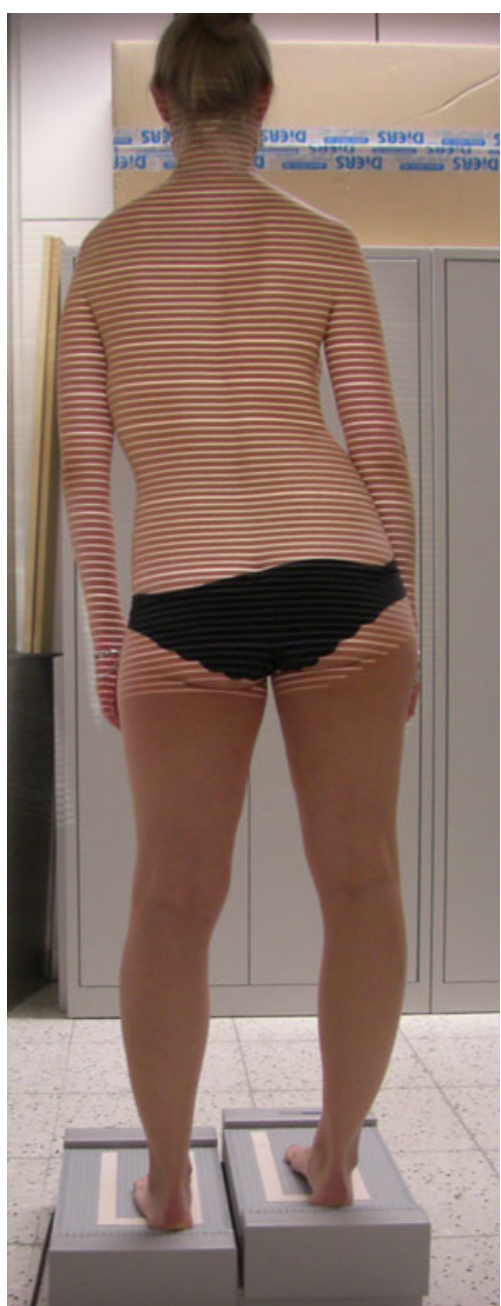

Fig. 7 The figure shows a female test subject during measurements standing on the simulation platform, which was elevated on the right leg side. The elevation of the platform led to a pelvic obliquity, with an elevation of the iliac crest on the long leg side. This pelvic obliquity resulted in a lateral deviation and convexity of the spine towards the short leg side

changes in pelvic position [5]. In that study we measured a mean pelvic obliquity of $4.20 \mathrm{~mm}$, which correlates closely with the mean value of $4.23 \mathrm{~mm}$ that we determined in this present study [5]. Froh et al. [18], Knutson [19] and ten Brinke et al. [20] all have radiologically measured pelvic obliquity reporting values ranging from 5.2 to $6.5 \mathrm{~mm}$. These results do not significantly differ $(p>0.05)$ from the results measured in our study for pelvic obliquity. However, the results are not directly comparable because of different imaging techniques that were used.

A mean pelvic torsion of $1.95^{\circ}$ was determined for subjects in the neutral standing position. This result does not differ statistically $(p>0.05)$ from that measured in our previous study, where a mean pelvic torsion of $0.35^{\circ}$ was determined. In a previous study, Young et al. have shown a significant increase in the pelvic torsion, when increasing the simulated LLIs. However, Drerup et al. [21] demonstrated that these changes are non-proportional and hard to predict because of complex interactions in the kinematic chain of the lower extremity and the pelvis. We were able to show a correlation between LLIs and changes of the pelvic position in LLIs even up to $60 \mathrm{~mm}$ and in addition we could show that LLIs of $20 \mathrm{~mm}$ and more lead to significant changes in the pelvic obliquity and torsion. The results of the parameter pelvic inclination showed that an increase in LLI also leads to an increase in pelvic inclination, which is significant $(p<0.05)$ with LLIs of $50 \mathrm{~mm}$ and above on both sides. These findings suggest that a further compensation mechanism of the kinematic chain, besides pelvic obliquity and torsion, exists. It seems to be a tilt of the pelvis as indicated by an increase in pelvic inclination. We also found a significant difference for the pelvic inclination between sexes. This difference was present in the neutral standing position and for all simulated LLIs. These results are in contrast to the findings of Janssen et al. [22] who found a significant difference in the spinal alignment between sexes, but not in particular for the pelvic inclination. However, the response of the pelvic inclination to the LLIs was similar between women and men, suggesting the same compensation mechanisms of the pelvis to LLIs in static conditions.

Encouraged by the results of our previous study, we became interested in the amount of LLI that would lead to significant changes in spinal parameters [5]. Jones et al. [23] described the relationship between LLI and scoliosis as complex. Irving found in 1991 [24], a relationship between an unlevelness of the sacral base and the degree of the resulting lumbar scoliosis. Despite extensive research efforts, there are still controversies about changes of the spine due to LLIs, especially about the amount of LLI that will create alterations in spinal parameters. The results of our study show for the first time that LLIs of $20 \mathrm{~mm}$ on the left and $30 \mathrm{~mm}$ on the right side lead to significant changes in the spine.

Our experience with this technique has shown that a surface rotation over $5^{\circ}$ is significantly associated with scoliotic changes (data not shown). Young et al. [21] stated that by placing a lift under one foot the lateral flexion of the spine increased. In addition they have noted that due to this increased lateral flexion, the lumbar vertebrae have a tendency to undergo a conjunct rotation. Our results confirmed a significant increase of the lateral flexion of the spine due to a $20 \mathrm{~mm}$ lift on the left and a $30 \mathrm{~mm}$ lift on the right side and that these changes were still present in higher LLIs of up to $60 \mathrm{~mm}$ (Fig. 6).

We have found a tendency to decreasing kyphotic angles with an increase in LLIs, which was not statistically significant $(p>0.05)$. A possible explanation might be that we only measured acute changes and not longstanding 
Table 1 Mean and SD for the sagittal spine parameters kyphotic and lordotic angles in degrees

\begin{tabular}{|c|c|c|c|c|}
\hline \multirow[t]{2}{*}{ Platform height $(\mathrm{mm})$} & \multicolumn{2}{|c|}{ Kyphotic angle (SD) } & \multicolumn{2}{|c|}{ Lordotic angle (SD) } \\
\hline & Females & Males & Females & Males \\
\hline-60 & $47.36(9.81)$ & $46.42(14.29)$ & $44.68(8.29)$ & $35.43(8.89)$ \\
\hline-50 & 48.89 (10.36) & $48.18(14.15)$ & $45.22(8.62)$ & $36.38(8.45)$ \\
\hline-40 & $49.98(9.95)$ & $50.38(11.11)$ & $45.08(8.26)$ & $36.62(6.92)$ \\
\hline-30 & $49.31(9.07)$ & $51.08(10.98)$ & $43.84(8.25)$ & $36.15(6.41)$ \\
\hline-20 & $50.54(9.36)$ & $52.37(10.8)$ & $44.13(8.39)$ & $34.93(7.59)$ \\
\hline-10 & $50.44(9.63)$ & $52.39(9.36)$ & $43.29(8.06)$ & $35.67(7.4)$ \\
\hline 0 & $48.94(8.51)$ & $51.97(10.26)$ & $41.96(8.42)$ & $33.52(8.04)$ \\
\hline 10 & $50.2(8.49)$ & $52.45(10.33)$ & $43.08(8.37)$ & $34.88(7.44)$ \\
\hline 20 & $49.84(8.64)$ & $52.27(9.68)$ & $43.8(8.05)$ & $35.29(7.16)$ \\
\hline 30 & $49.66(8.92)$ & $51.1(10.44)$ & $44.22(7.82)$ & $35.47(7.71)$ \\
\hline 40 & $49.56(9.04)$ & $51.05(10.53)$ & $44.39(7.09)$ & $35.69(6.72)$ \\
\hline 50 & $48.47(9.72)$ & $48.12(13.38)$ & $43.77(7.46)$ & $35.33(7.02)$ \\
\hline 60 & $46.46(9.94)$ & $46.08(14.23)$ & $43.58(7.27)$ & $33.93(7.27)$ \\
\hline
\end{tabular}

There were no significant differences between the different simulated platform heights and changes in these parameters, but we found differences between the lordotic angles of females and males with women having significant higher lordotic angles

ones. A further explanation could be that the range for "normal" kyphotic angles seems to be larger, as reflected by the high standard deviations of up to $10^{\circ}$, and therefore statistically significant changes were not found.

In our study there was a difference in changes of the pelvic and spinal parameters between the left $(>20 \mathrm{~mm})$ and the right side $(>30 \mathrm{~mm})$ when inducing a LLI. A possible explanation for the differences between sides could be the dominant leg effect. In the upper extremities a left or right hand dominance is well documented [25] and it could be assumed that this also exists in the lower extremities [26]. A further explanation for the side differences could also be muscle imbalances or differences in range of motion of the joints [27].

Women had a significant higher lordosis than males. This was also noted by Lang-Tapia et al. [28] who stated that women seem to have a greater lumbar lordosis, possibly because of differences in vertebral shape and changes that occur during pregnancy. We did not find any significant gender differences in the kyphotic angle, which also was in accordance with Lang-Tapia et al. [28]. We also found no differences between females and males in the response to different LLIs. Therefore, we conclude that women and men react similarly to LLIs of up to $60 \mathrm{~mm}$.

A limitation of our study is that we examined acute changes of the leg length instead of long-standing leg length differences. We do think that further studies would be of interest to analyse the effects of long-standing LLIs on spinal posture and pelvic position, but until now there does not exist an evidence-based definition of long-standing LLIs (weeks, months, years?), which makes it not feasible to investigate. A limitation of our study is that the effects of
LLIs were only examined under static conditions, while the subjects were standing on a platform. In future studies, with the development of faster video rasterstereographic systems, a three-dimensional analysis of the here found changes under dynamic conditions would be of interest. However, it must be noted that the clinical diagnosis and treatment of pelvic obliquity still often takes place in a static, standing position with extended knees, and therefore we adopted and investigated this situation in our study.

\section{Conclusion}

For the first time the results of our study have shown that LLIs of more than $20 \mathrm{~mm}$ lead to significant changes in the spinal posture. We also found a significant correlation between LLIs and pelvic position. Further there was no difference found in the reaction to LLIs between women and men.

Acknowledgments No funds were received in support of this work. No benefits in any form have been or will be received from a commercial party related directly or indirectly to the subject of this manuscript.

Conflict of interest None.

\section{References}

1. Perttunen JR, Anttila E, Sodergard J, Merikanto J, Komi PV (2004) Gait asymmetry in patients with limb length discrepancy. Scand J Med Sci Sports 14(1):49-56 
2. Friberg O (1983) Clinical symptoms and biomechanics of lumbar spine and hip joint in leg length inequality. Spine (Phila $\mathrm{Pa} 1976$ ) 8(6):643-651

3. Giles LG, Taylor JR (1982) Lumbar spine structural changes associated with leg length inequality. Spine (Phila Pa 1976) 7(2): 159-162

4. Papaioannou T, Stokes I, Kenwright J (1982) Scoliosis associated with limb-length inequality. J Bone Joint Surg Am 64(1):59-62

5. Betsch M, Wild M, Grosse B, Rapp W, Horstmann T (2011) The effect of simulating leg length inequality on spinal posture and pelvic position: a dynamic rasterstereographic analysis. Eur Spine J 21(4):691-697. doi:10.1007/s00586-011-1912-5

6. Drerup B, Hierholzer E (1987) Movement of the human pelvis and displacement of related anatomical landmarks on the body surface. J Biomech 20(10):971-977

7. Drerup B, Hierholzer E (1987) Automatic localization of anatomical landmarks on the back surface and construction of a body-fixed coordinate system. J Biomech 20(10):961-970

8. Beaudoin L, Zabjek KF, Leroux MA, Coillard C, Rivard CH (1999) Acute systematic and variable postural adaptations induced by an orthopaedic shoe lift in control subjects. Eur Spine J 8(1):40-45

9. Langer S (1976) Structural leg shortage. A case report. J Am Podiatry Assoc 66(1):38-40

10. Blustein SM, D'Amico JC (1985) Limb length discrepancy. Identification, clinical significance, and management. J Am Podiatr Med Assoc 75(4):200-206

11. Betsch M, Wild M, Jungbluth P, Hakimi M, Windolf J, Haex B, Horstmann T, Rapp W (2011) Reliability and validity of 4D rasterstereography under dynamic conditions. Comput Biol Med 41(6):308-312. doi:10.1016/j.compbiomed.2011.03.008

12. Hackenberg L, Hierholzer E, Liljenqvist U (2002) Accuracy of rasterstereography versus radiography in idiopathic scoliosis after anterior correction and fusion. Stud Health Technol Inform 91: 241-245

13. Hackenberg L, Liljenqvist U, Hierholzer E, Halm H (2000) Scanning stereographic surface measurement in idiopathic scoliosis after VDS (ventral derotation spondylodesis). Z Orthop Ihre Grenzgeb 138(4):353-359. doi:10.1055/s-2000-10162

14. Goh SPR, Leedman PJ, Singer KP (1999) Rasterstereographic analysis of the thoracic sagittal curvature: a reliability study. J Muscoskel Res 3(2):137-142

15. Crawford RJ, Price RI, Singer KP (2009) The effect of interspinous implant surgery on back surface shape and radiographic lumbar curvature. Clin Biomech (Bristol, Avon) 24(6):467-472. doi:10.1016/j.clinbiomech.2009.04.003
16. Goh S, Price RI, Leedman PJ, Singer KP (2000) A comparison of three methods for measuring thoracic kyphosis: implications for clinical studies. Rheumatology (Oxford) 39(3):310-315

17. Drerup B, Ellger B, Hierholzer E, Meyer zu Bentrup FM (2001) Functional rasterstereographic images. A new method for biomechanical analysis of skeletal geometry. Orthopade 30(4): 242-250

18. Froh R, Yong-Hing K, Cassidy JD, Houston CS (1988) The relationship between leg length discrepancy and lumbar facet orientation. Spine (Phila Pa 1976) 13(3):325-327

19. Knutson GA (2005) Anatomic and functional leg-length inequality: a review and recommendation for clinical decisionmaking. Part I, anatomic leg-length inequality: prevalence, magnitude, effects and clinical significance. Chiropr Osteopat 13:11

20. ten Brinke A, van der Aa HE, van der Palen J, Oosterveld F (1999) Is leg length discrepancy associated with the side of radiating pain in patients with a lumbar herniated disc? Spine (Phila Pa 1976) 24(7):684-686

21. Young RS, Andrew PD, Cummings GS (2000) Effect of simulating leg length inequality on pelvic torsion and trunk mobility. Gait Posture 11(3):217-223

22. Janssen MM, Drevelle X, Humbert L, Skalli W, Castelein RM (2009) Differences in male and female spino-pelvic alignment in asymptomatic young adults: a three-dimensional analysis using upright low-dose digital biplanar X-rays. Spine (Phila Pa 1976) 34(23):E826-E832. doi:10.1097/BRS.0b013e3181a9fd85

23. Jones KB, Sponseller PD, Hobbs W, Pyeritz RE (2002) Leglength discrepancy and scoliosis in Marfan syndrome. J Pediatr Orthop 22(6):807-812

24. Irvin RE (1991) Reduction of lumbar scoliosis by use of a heel lift to level the sacral base. J Am Osteopath Assoc 91(1):34, $37-44$

25. Frayer DW, Lozano M, Bermudez de Castro JM, Carbonell E, Arsuaga JL, Radovcic J, Fiore I, Bondioli L More than 500,000 years of right-handedness in Europe. Laterality 1-19. doi: 10.1080/1357650X.2010.529451

26. DeVita P, Hong D, Hamill J (1991) Effects of asymmetric load carrying on the biomechanics of walking. J Biomech 24(12): 1119-1129

27. Hesse S, Reiter F, Jahnke M, Dawson M, Sarkodie-Gyan T, Mauritz KH (1997) Asymmetry of gait initiation in hemiparetic stroke subjects. Arch Phys Med Rehabil 78(7):719-724

28. Lang-Tapia M, Espana-Romero V, Anelo J, Castillo MJ (2011) Differences on spinal curvature in standing position by gender, age and weight status using a noninvasive method. J Appl Biomech 27(2):143-150 